\title{
Obsessional cliques: a semantic characterization of bounded time complexity
}

\author{
Olivier Laurent ${ }^{* \dagger}$ \\ Preuves Programmes Systèmes \\ CNRS - Paris 7 \\ E-mail: Olivier.Laurentepps.jussieu.fr
}

\author{
Lorenzo Tortora de Falco ${ }^{* \ddagger}$ \\ Dipartimento di filosofia \\ Roma III \\ E-mail: tortora@uniroma3.it
}

\begin{abstract}
We give a semantic characterization of bounded complexity proofs. We introduce the notion of obsessional clique in the relational model of linear logic and show that restricting the morphisms of the category $\mathcal{R E} \mathcal{L}$ to obsessional cliques yields models of ELL and SLL. Conversely, we prove that these models are relatively complete: an $\mathrm{LL}$ proof whose interpretation is an obsessional clique is always an ELL/SLL proof. These results are achieved by introducing a system of ELL/SLL untyped proof-nets, which is both correct and complete with respect to elementary/polynomial time complexity.
\end{abstract}

\section{Introduction}

It is widely acknowledged that computational complexity is a central topic in modern science: the famous $P$ versus NP question appears in several (apparently) unrelated areas of pure and applied mathematics and theoretical computer science. Behind it, lies our capability of handling resources and of keeping control on their use: a crucial point for the contemporary computer scientist. The difficulty of the problem seems related to our yet incomplete understanding of the very nature of polynomial time.

Many of the scientists who tried to fathom the secrets of polytime produced alternative definitions of computability within a given bound, without any explicit reference to the bound itself. Usually, these characterizations are driven by the cultural background of their authors, who expect some help from the techniques developed in their own fields of research. This approach (having among his forerunners Kalmar and his inductive definition of the class of elementary recursive functions dating back to 1943) involves various branches of mathematical logic: (finite) model theory, recursion theory, proof-theory. Let us quote

\footnotetext{
* Supported by the project "Coop. Italia/France CNR-CNRS n ${ }^{\circ} 19188$ ".

†Supported by the French project "ANR JC05_43380 No-Cost".

"Supported by the Italian project "FOLLIA".
}

some of the main contributions: Fagin's characterization of NPTIME [12] on the model-theoretical side, limitations of the recursion schemata $[6,4]$ on the recursion-theoretical side, propositional proof complexity [7] and Buss' Bounded Arithmetic [5] on the proof-theoretical side. These results contributed to the birth of a new research area, now called Implicit Computational Complexity.

Taking the "Curry-Howard looking glass" (a proof is a program whose execution corresponds to applying the cut-elimination procedure to the proof), the approach to complexity is based on the idea that the expressive power of a logical system is the complexity of its cutelimination/normalization procedure. Much work has been done in the framework of typed $\lambda$-calculus (for example [18]): roughly speaking, some limitations on the way $\lambda$-terms "communicate" allow to keep normalization polynomial. From the (strictly) logical point of view, the introduction of Linear Logic (LL [13]) was an important step: $\mathrm{LL}$ is a refinement of intuitionistic and classical logics characterized by the introduction of new connectives (the exponentials) which give a logical status to the operations of erasing and copying (corresponding to the structural rules of intuitionistic and classical logics). This shed a new light on the duplication process responsible of the "explosion" of the size (and time) during the cut-elimination procedure, and led to a first result in [16]. But, in this Bounded Linear Logic, polynomials appear explicitly. A notable breakthrough is Girard's Light Linear Logic (LLL [15]): a very careful handling of LL's exponentials allows the author to keep enough control on the duplication process. He proves that a function $f$ is representable in LLL if and only if $f$ is polytime. More recently, other "light systems" have been introduced by Asperti and Roversi [1, 2], Danos and Joinet [10], Lafont [17] and others: several simplifications are proposed and suggest that LLL is only one among the possible solutions (rather a research theme than a logical system).

Since the beginning [15], light systems are presented as subsystems of LL obtained by restricting the use of the exponentials: some principles (formulas) provable in LL do 
not hold in light systems. However, a more geometric perspective on light logic is possible. It comes from the introduction of proof-nets, a geometric way of representing computations; actually one of the most important consequences of the logical status given by $L L$ to the structural rules. Light proofs have been presented in [15] as proof-nets and the geometric view was corroborated by a crucial property of light proof(-net)s: stratification. Computations are performed layer by layer: the so-called depth of the proofnet (a geometric parameter) is invariant during computation. This viewpoint was stressed in [10], where the authors give a geometric characterization of those proof-nets with an elementary cut-elimination: the system is not modified and a global condition on the graph representation of proofs allows to isolate the "elementary" ones. A similar work for LLL has been done in [20]. We feel these results are little steps towards a more abstract vision of bounded complexity.

Among the questions and problems arisen from [15], the quest of a denotational semantics (a semantics of proofs in logical terms, or more generally a model) suitable for light systems is maybe the main one: hopefully, such a semantics will inspire a new mathematical point of view on the nature of polytime. Indeed, the general goal of denotational semantics is to give a "mathematical" counterpart to syntactical devices such as proofs and programs, thus bringing to the fore their essential properties. It maps the concrete syntactical objects to an algebraic, geometric, categorical, ... description, which stresses basic invariants and, sometimes, eventually results in improvements of the syntax: LL itself comes from a denotational model of second order intuitionistic logic. The basic pattern is to associate with every formula/type some structure and with every proof/program of the formula/type an element of the structure (called its interpretation). Clearly, interpreting LL proofs allows to interpret proofs of a given light system but gives no information on the "lightness" of proofs; the point is to find a denotational semantics of a light system which is not a denotational semantics of LL. To our knowledge only two proposals [21, 3] have been made up to now. While technically rather different ([21] is based on game semantics and [3] on coherent semantics), the two works are similar in spirit: the structures (games, coherent spaces) associated with logical formulas are modified, so that the principles valid in LL but not in the chosen light system do not hold in the semantics. One can also mention the works by M. Hofmann et al. introducing realisability models where resource-boundedness is explicitly required on realisers (see [8] for example).

We propose a new approach to the semantics of proofs of light systems. Following the same spirit of the previously mentioned geometric perspective on light logic, instead of modifying the structures associated with logical formulas, we look for a property of the elements of the structures (the interpretations of proofs) characterizing those elements which can interpret polytime proofs. For this purpose, nothing like full completeness/surjectivity ("every element of the structure interpreting a provable formula is the interpretation of a proof") is required (by the way, in the models we consider, this property fails). The idea we develop here is to choose a light system and a denotational model of LL, and to prove that an LL proof $\pi$ is a proof of the chosen light system if and only if the interpretation $\llbracket \pi \rrbracket$ of $\pi$ satisfies some given (semantic) property. In order to obtain a model of the light system, we then need to check that the property has a "good behaviour" (mainly, it must be stable with respect to composition). Notice that in case we succeed, we get (much) more than a model: a relatively complete model, whose morphisms are exactly the ones of the $L L$ model satisfying the semantic property. Of course, the "quality" of the model will depend on the "quality" of the semantic property. The property we propose is obsessionality: it is very simple and rather natural; however (as explained in the conclusion) it does not say much on the elements which are not interpretations of proofs.

In this work, we choose as light logical systems ELL for elementary time and SLL for polynomial time, as model the relational one (but coherent semantics would also do). These choices are discussed in the conclusion. We introduce the notion of obsessional clique: a clique is obsessional when it is closed with respect to the (appropriate) action of the monoid $\mathbb{N}^{*}$ (definition 3 ). We prove that an LL proof $\pi$ is a proof of ELL/SLL if and only if $\llbracket \pi \rrbracket$ is obsessional (with the appropriate variant for ELL/SLL) and that obsessionality is a property with a "good behaviour" (as mentioned above).

One of the (striking) features of obsessional cliques is the absence of any explicit reference to stratification: this seems to be an interesting achievement, and a small step towards a truly semantic view of bounded complexity. What can be certainly affirmed is that our analysis is not syntax-driven, but a natural refinement of tools introduced for other purposes. Technically speaking, the starting point of this work is the notion of obsessional experiment introduced in [25]: it was used to "rebuild" an LL proof from its interpretation in (relational or coherent) semantics. In general this is not possible: there are different LL proofs with the same semantics as it is shown in [25]. However, the relational (resp. coherent) interpretation of a proof (and more precisely the result of an obsessional experiment of the proof) is enough to rebuild that part of the proof allowing to distinguish ELL (resp. SLL) proofs from the others.

Roughly speaking, obsessional cliques have been introduced following the idea that, in case the clique is the interpretation of a proof-net, obsessional experiments "have all to be available". It turned out (and this was not a priori obvious) that obsessional cliques compose and yield a model of propositional ELL (resp. SLL): the category 
$\mathcal{O} \mathcal{R} \mathcal{L}$ (resp. $\mathcal{S} \mathcal{R} \mathcal{E} \mathcal{L}$ ), as proved in section 2. One could prove a relative completeness theorem in the style of theorem 4 in a propositional framework, but one would not obtain a "semantic characterization of bounded time complexity": the considered fragments are not expressive enough to represent all elementary (resp. polynomial) computations. In ELL/SLL, the second order quantifiers are necessary to encode polytime computations [19]. Instead of dealing with the semantics of second order quantifiers (which is delicate), we moved from the typed to the untyped framework, thus avoiding the difficulty, in the spirit of [11]. The complexity of the cut-elimination procedures of $[15,17]$ does not depend on the complexity of cut-formulas, but only on the graph representation of the proofs as proof-nets. The elementary/polynomial complexity bound can thus be straightforwardly extended from the typed to the untyped case. Conversely, whatever can be represented in a typed framework can also be represented in an untyped one: simply forget types!

In the spirit of the semantics of $\lambda$-calculus (a model of the untyped $\lambda$-calculus is a model of the typed $\lambda$-calculus with a reflexive object), we decided to first present the typed framework (section 2) and move later to the untyped one. Section 3 is devoted to introduce (untyped) nets, an extension of Danos-Regnier's untyped proof-nets. In section 4, following [10], we define untyped ELL and SLL nets in geometric terms. Finally, we prove the main result of the paper in section 5: we introduce a space for "untyped obsessionality" in the spirit of the relational model and of the other models of the untyped $\lambda$-calculus. We adapt the notion of experiment of a proof-net (introduced in [13]) to define a model of our untyped nets and we prove the relative completeness theorem (theorem 4).

\section{Typed case}

After a short presentation of the two systems ELL and SLL, we are going to extend the relational model of linear logic with an action of $\mathbb{N}^{*}$ (the set of positive integers) on sets in order to define denotational models of these two systems.

\subsection{Second order ELL and SLL}

We consider the two subsystems ELL [15] and SLL [17] of $L L$ which respectively correspond to elementary time and polynomial time complexities. See figures 1 and 2 for ELL, and figures 1 and 3 for SLL.

In ELL, integers are represented by proofs of $\mathbb{U}=$ $\forall X ?\left(X \otimes X^{\perp}\right) \not \gamma !\left(X^{\perp} \not \gamma X\right)$, and functions are represented by proofs of $\vdash \mathbb{U}^{\perp}, !^{p} \mathbb{U}$. As shown in $[15,10]$, representable functions in ELL are exactly elementary time functions.
In SLL, binary integers are represented by proofs of $\mathbb{W}=\forall X ?\left(\mathbb{B} \otimes X \otimes X^{\perp}\right) \not \gamma X^{\perp} \not \gamma X$ where $\mathbb{B}=\forall X X^{\perp} \not \gamma$ $X^{\perp} \chi(X \otimes X)$, and predicates over integers are represented by proofs of $\vdash \mathbb{W} \perp, \ldots, \mathbb{W} \perp, \mathbb{B}$ which do not contain any ? $m$-rule (for the application of a predicate to an argument in $\mathbb{W}$, we first have to duplicate it the appropriate number of times). As shown in [19], representable predicates in SLL are exactly polynomial time predicates.

\subsection{Obsessional relational model}

Notations. If $E$ is a set, we denote by $\mathcal{M}_{f}(E)$ the set of finite multisets of elements of $E$ (i.e. the free commutative monoid generated by $E$ ). [] is the empty multiset and + is the commutative monoid law. If $x_{1}, \ldots, x_{n}$ are elements of $E$ and $k_{1}, \ldots, k_{n}$ are natural numbers, $\left[k_{1} x_{1}, \ldots, k_{n} x_{n}\right]$ is the multiset containing $k_{1}$ copies of $x_{1}, \ldots, k_{n}$ copies of $x_{n}$.

The category $\mathcal{R E} \mathcal{L}$ of sets and relations is one of the simplest models of LL. The interpretation of connectives is given by: $1=\perp=\{\star\}, \otimes=\not \gamma=\times$ and $!=?=\mathcal{M}_{f}$.

In the spirit of coherent spaces, we call cliques of $A$ (denoted $c \sqsubset A$ ) the subsets of $A$.

\section{Definition 1 ( $\mathbb{N}$-set)}

A $\mathbb{N}$-set is given by a set $A$ and a function $(k, a) \mapsto a^{(k)}$ from $\mathbb{N}^{*} \times A$ to $A$, called the action, which is an action of the monoid $\left(\mathbb{N}^{*}, \cdot, 1\right)$ on $A$ (that is $a^{(1)}=a$ and $a^{\left(k k^{\prime}\right)}=$ $\left.\left(a^{(k)}\right)^{\left(k^{\prime}\right)}\right)$.

The constructions of $\mathbb{N}$-sets are obtained from the corresponding constructions of sets and the actions are built in the following way:

- on $1=\{\star\}$, we use the only possible action

- the action on $A \times B$ is given by $(a, b)^{(k)}=\left(a^{(k)}, b^{(k)}\right)$

- if $t \in \mathbb{N}$, we define $!_{t} A$ (resp. ${ }_{t} A$ ) as the $\mathbb{N}$-set with underlying set $\mathcal{M}_{f}(A)$ and the action on $!_{t} A$ (resp. ${ }_{t} A$ ) is given by:

$$
\left[a_{1}, \ldots, a_{n}\right]^{(k)}= \begin{cases}{\left[a_{1}^{(k)}, \ldots, a_{n}^{(k)}\right]} & \text { if } n \leq t \\ {\left[k a_{1}^{(k)}, \ldots, k a_{n}^{(k)}\right]} & \text { if } n>t\end{cases}
$$

Definition 2 (Category $\mathbb{N} \mathcal{R} \mathcal{E} \mathcal{L}$ )

The category $\mathbb{N} \mathcal{R} \mathcal{E} \mathcal{L}$ is given by:

- objects: $\mathbb{N}$-sets

- morphisms: $\mathbb{N} \mathcal{R} \mathcal{E} \mathcal{L}(A, B)$ is the set of cliques of $A \times B$

The categories $\mathcal{R E} \mathcal{L}$ and $\mathbb{N} \mathcal{R E} \mathcal{L}$ are equivalent categories, thus $\mathbb{N} \mathcal{R} \mathcal{E} \mathcal{L}$ is a model of $L L$.

We are now able to define the key notion of this paper.

\section{Definition 3 (Obsessional clique)}

Let $A$ be a $\mathbb{N}$-set, a clique $c$ is obsessional if $\forall a \in c, \forall k \in$ $\mathbb{N}^{*}, a^{(k)} \in c$.

\section{Proposition 1 (Category $\mathcal{O} \mathcal{R} \mathcal{E} \mathcal{L}$ )}

The category $\mathcal{O} \mathcal{R} \mathcal{L} \mathcal{L}$ is the subcategory of $\mathbb{N} \mathcal{R} \mathcal{E} \mathcal{L}$ with all objects and only obsessional cliques as morphisms. 
The $\star$-autonomous structure of $\mathbb{N} \mathcal{R E} \mathcal{L}$ respects obsessionality: the cliques $(A \times B) \times C \simeq A \times(B \times C)$, $A \times B \simeq B \times A$ and $A \times 1 \simeq A$ are all obsessional, and if $x \sqsubset A \times C$ and $y \sqsubset B \times D$ are obsessional then $x \times y=\{((a, b),(c, d)) \mid(a, c) \in x \wedge(b, d) \in y\} \sqsubset$ $(A \times B) \times(C \times D)$ is obsessional.

Concerning the exponential structure:

- If $x \sqsubset \sqsubset A \times B$ is obsessional, $! x=$ $\left\{\left(\left[a_{1}, \ldots, a_{n}\right],\left[b_{1}, \ldots, b_{n}\right]\right) \quad \mid \forall 1 \leq i \leq n,\left(a_{i}, b_{i}\right) \in\right.$ $x\} \sqsubset !_{t} A \times !_{t} B$ is obsessional for any $t$.

- $\left\{\left(\left(\left[a_{1}, \ldots, a_{n}\right],\left[b_{1}, \ldots, b_{n}\right]\right),\left[\left(a_{1}, b_{1}\right), \ldots,\left(a_{n}, b_{n}\right)\right]\right)\right\} \sqsubset$ $\left(!_{t} A \times !_{t} B\right) \times !_{t}(A \times B)$ is obsessional for any $t$.

- $\{(\star,[n \star]) \mid n \in \mathbb{N}\} \sqsubset 1 \times !_{t} 1$ is obsessional for any $t$.

- $\left\{\left(\left[a_{1}, \ldots, a_{n}\right],\left(a_{1}, \ldots, a_{n}\right)\right)\right\} \sqsubset !_{t} A \times \prod_{n} A$ is obsessional for $t \geq n$. In particular if $n=0,\{([], \star)\} \sqsubset$ $!_{t} A \times 1$ is obsessional for any $t$.

- $\{(\mu+\nu,(\mu, \nu))\} \sqsubset !_{0} A \times\left(!_{0} A \times !_{0} A\right)$ is obsessional. Conversely, obsessionality allows to "refute" the usual representation of various principles:

- contraction is not obsessional in $!_{t} A \times\left(!_{t} A \times !_{t} A\right)$ as soon as $t>0$

- digging is not obsessional in $!_{t} A \times !_{t} !_{t} A$ for any $t$

- dereliction is not obsessional in $!_{0} A \times A$

\section{Theorem 1}

$\mathcal{O R E} \mathcal{L}$ is a model of propositional (additive-free) ELL, interpreting every formula of type $! A$ by $!_{0} A$.

Proof. We have shown that the required constructions preserve obsessionality, and using the faithful functor $U$ into $\mathcal{R} \mathcal{E} \mathcal{L}$ given by $U\left(A,{ }_{-}{ }^{-}\right)=A$ and $U(x)=x$ if $x \sqsubset A \times B$, we can show that all the required diagrams commute.

\section{Definition 4 (Category $\mathcal{S} \mathcal{R} \mathcal{L}^{1}$ )}

The category $\mathcal{S R \mathcal { E }} \mathcal{L}$ is given by:

- objects: an object is a set with a family of actions indexed by $\mathbb{N}$ giving it $\mathbb{N}$-set structures $\left(A_{n}\right)_{n \in \mathbb{N}}$

- morphisms: elements of $\mathcal{S} \mathcal{R} \mathcal{E} \mathcal{L}\left(\left(A_{n}\right)_{n \in \mathbb{N}},\left(B_{n}\right)_{n \in \mathbb{N}}\right)$ are cliques $x$ of $A \times B$ such that there exists some $t \in \mathbb{N}$ with for any $n \geq t, x$ is an obsessional clique of $A_{n} \times B_{n}$

Any $\mathbb{N}$-set $A$ can be turned into an object in $\mathcal{S R \mathcal { L }} \mathcal{L}$ by choosing all the actions of the family to be the action of $A$. In the same spirit, constructions on objects of $\mathbb{N} \mathcal{R} \mathcal{L}$ can be turned into constructions on objects of $\mathcal{S R \mathcal { E }} \mathcal{L}$ by applying them for each $n$. As specific constructions, we define: $!\left(A_{n}\right)_{n \in \mathbb{N}}=\left(!_{n} A_{n}\right)_{n \in \mathbb{N}}$ and $?\left(A_{n}\right)_{n \in \mathbb{N}}=\left(?_{n} A_{n}\right)_{n \in \mathbb{N}}$.

\section{Theorem 2}

$\mathcal{S R \mathcal { L }}$ is a model of propositional (additive-free) SLL.

Proof. As for ELL, using a forgetful functor into $\mathcal{R E} \mathcal{L}$.

\footnotetext{
${ }^{1}$ In a parallel work, B. Redmond [22] is developing a categorical interpretation of SLL. It seems that $\mathcal{S R \mathcal { E }} \mathcal{L}$ can be obtained by applying one of his constructions. The relationships has to be investigated further
}

\section{Nets}

We extend Danos-Regnier's notion of untyped proofnets $[9,23]$ to a more liberal notion which contains the direct translation of second order multiplicative exponential LL.

\section{Definition 5 (Proof-structure)}

A proof-structure is a finite directed acyclic graph ${ }^{2}$ whose nodes are defined together with an arity and a coarity, i.e. a given number of incident edges called the premisses of the node and a given number of emergent edges called the conclusions of the node. The valid nodes are the following:

\begin{tabular}{|l|c|c|c|c|c|}
\hline nodes & ax & cut & $\otimes, \mathcal{X}, ? c$ & $1, \perp, ? w$ & $!, ? d, ? p$ \\
\hline arity & 0 & 2 & 2 & 0 & 1 \\
coarity & 2 & 0 & 1 & 1 & 1 \\
\hline
\end{tabular}

We allow edges with a source but no target, they are called conclusions of the proof-structure.

Some edges are labelled with the ? symbol according to the following rules:

- conclusions of $a x, 1, \perp, \otimes, \ngtr$ and !-nodes are not labelled,

- conclusions of $? d, ? c, ? w$ and ? $p$-nodes are ?-labelled,

- premisses of ?c and ?p-nodes are ?-labelled.

Moreover, a proof-structure $\mathcal{R}$ has to satisfy the following two properties:

- !-box condition:

- with every !-node $n$ is associated a subgraph $B^{n}$ of $\mathcal{R}$, such that one of the conclusions of $B^{n}$ is the conclusion of $n$ and every other conclusion of $B^{n}$ (there might be no other conclusion) is the conclusion of a ?p-node. $B^{n}$ is called a box and it is represented by a rectangular frame. $n$ is called the main door of $B^{n}$

- with every ? $p$-node $p$ is associated the box $B^{n}$ of some !-node $n$, such that the conclusion of $p$ is conclusion of $B^{n}$. The node $p$ is called an auxiliary door of $B^{n}$.

- nesting condition: two boxes are either disjoint or included one in the other.

We will often speak of a box, a node or an edge of a proof-structure $\mathcal{R}$ contained in a box $B$ of $\mathcal{R}$. In case of nodes, we will not consider the doors of $B$ as nodes contained in $B$. The depth of an edge is the number of boxes containing it. The depth of $\mathcal{R}$ is the maximal depth of its edges.

The ? labels are the only kind of typing we consider. It is somehow the heart of linear logic: the distinction between duplicable/erasable formulas and the others. The reader should notice that these labels introduce constraints

\footnotetext{
${ }^{2}$ When drawing a proof-structure we represent edges oriented up-down so that we may speak of moving upwardly or downwardly in the graph, and of nodes or edges "above" or "under" a given node/edge.
} 


$$
A::=X\left|X^{\perp}\right| 1|\perp| A \otimes A|A \not \supset A| ! A|? A| \forall X A \mid \exists X A
$$

Figure 1. Second order LL formulas.

$$
\begin{aligned}
& \frac{\vdash \Gamma, A \quad \vdash \Delta, A^{\perp}}{\vdash \text { 制 }} \text { cut }
\end{aligned}
$$

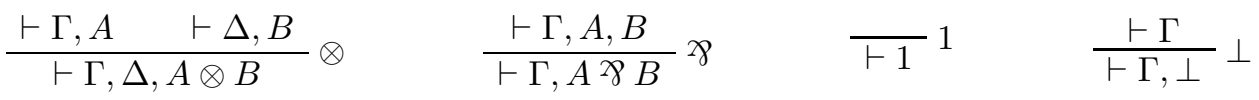

$$
\begin{aligned}
& \frac{\vdash \Gamma, A}{\vdash ? \Gamma, ! A} ! f \quad \frac{\vdash \Gamma, ? A, ? A}{\vdash \Gamma, ? A} ? c \quad \frac{\vdash \Gamma}{\vdash \Gamma, ? A} ? w \\
& \frac{\vdash \Gamma, A}{\vdash \Gamma, \forall X A} \forall \quad(X \notin \Gamma) \quad \frac{\vdash \Gamma, A\left[\left[^{B} / X\right]\right.}{\vdash \Gamma, \exists X A} \exists
\end{aligned}
$$

Figure 2. ELL rules.

$$
\begin{aligned}
& \frac{\vdash \Gamma, A \quad \vdash \Delta, A^{\perp}}{\vdash A, A^{\perp}} \text { cut } \\
& \frac{\vdash \Gamma, A \quad \vdash \Delta, B}{\vdash \Gamma, \Delta, A \otimes B} \otimes \quad \frac{\vdash \Gamma, A, B}{\vdash \Gamma, A \not \gamma B} \not 2 \quad \frac{\vdash \Gamma}{\vdash 1} 1 \\
& \frac{\vdash \Gamma, A}{\vdash ? \Gamma, ! A} ! f \quad \frac{\vdash \Gamma, A, \ldots, A}{\vdash \Gamma, ? A} ? m \\
& \frac{\vdash \Gamma, A}{\vdash \Gamma, \forall X A} \forall(X \notin \Gamma) \quad \frac{\vdash \Gamma, A\left[\left[^{B} / X\right]\right.}{\vdash \Gamma, \exists X A} \exists
\end{aligned}
$$

Figure 3. SLL rules.
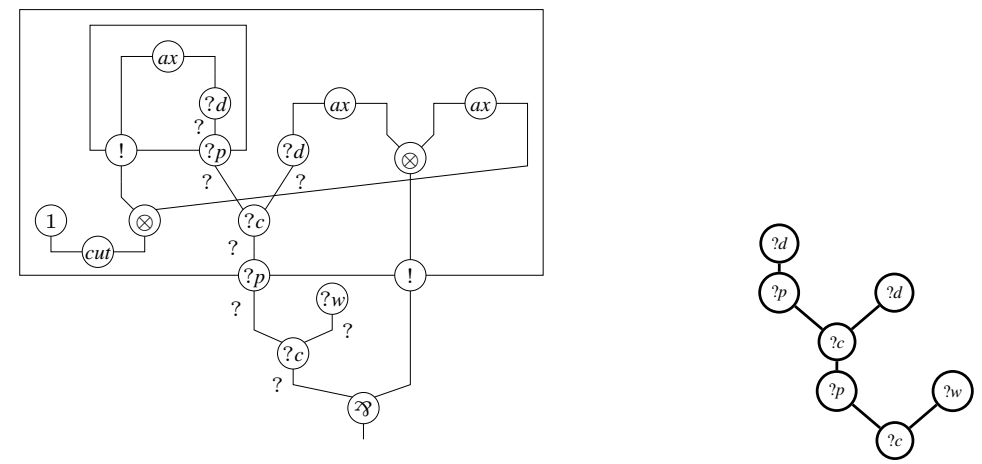

Figure 4. A (normal) net, the (maximal) exponential tree of the left premisse of the 8 -node and the associated exponential bundle of branches. 
on proof-structures: for example the conclusion of an $a x$ node cannot be the premise of a ?c-node.

\section{Definition 6 (Net)}

Let $\mathcal{R}$ be a proof-structure and let $B_{1}, \ldots, B_{k}$ be the boxes of $\mathcal{R}$ with depth zero. With $\mathcal{R}$ is naturally associated an undirected graph $G_{\mathcal{R}}$ with a given set of pairs of edges:

- substitute for each box $B_{i}(1 \leq i \leq k)$, a node with the conclusions of the doors of the box as conclusions,

- keep the other 0-depth nodes and forget the orientation,

- associate with every $\not 2$-node (resp. ? $c$-node) of $\mathcal{R}$ with depth zero the (unordered) pair of its premisses.

A switching $S$ of $\mathcal{R}$ is the choice of an edge for every pair of $G_{\mathcal{R}}$. With each switching $S$ is associated a subgraph $S(\mathcal{R})$ of $G_{\mathcal{R}}$ : for every pair of $G_{\mathcal{R}}$, erase the edges of $G_{\mathcal{R}}$ which are selected by $S$.

We say that $\mathcal{R}$ is a net when:

- for every switching $S$ of $\mathcal{R}, S(\mathcal{R})$ is acyclic,

- for every box $B_{i}(1 \leq i \leq k)$, the proof-structure $\mathcal{R}_{i}$ contained in $B_{i}$ is a net.

Due to the weak conditions we put on the way nodes can be used, we cannot guarantee that we have a way to reduce all cuts. This is strongly different from what happens with Danos-Regnier's untyped proof-nets where a weak notion of typing is enough to ensure reducibility of any cut-node.

The two edges premisses of a cut-node are dual when:

- they are conclusions of a $\otimes$-node and of a $X$-node,

- they are conclusions of a 1 -node and of a $\perp$-node,

- one is conclusion of a !-node and the other one is ?labelled.

\section{Definition 7 (Deadlock)}

A cut-node of a net is a deadlock when the premisses of the cut-node are not dual edges and none of the two is the conclusion of an $a x$-node.

\section{Definition 8 (Cut-elimination)}

The reduction steps are defined as usual [13]:

- $a x$-step: this step applies when one of the premisses of the cut-node is the conclusion of an $a x$-node. In this case one erases as usual both the $c u t$-node and the $a x$-node ${ }^{3}$.

- $2 / \otimes$-step: for a cut between a 28 -node and a $\otimes$-node.

- $\perp / 1$-step: for a cut between a $\perp$-node and a 1-node.

- ?d-step: for a cut between a ?d-node and a !-node.

- ?w-step: for a cut between a ?w-node and a !-node.

- ?c-step: for a cut between a ?c-node and a !-node.

- ?p-step: for a cut between a ?p-node and a !-node.

\section{Proposition 2}

If the procedure of cut-elimination cannot be applied to the net $\mathcal{R}$, then every cut-node of $\mathcal{R}$ is a deadlock (in particular, $\mathcal{R}$ might be cut-free). We call normal such a net $\mathcal{R}$.

\footnotetext{
${ }^{3}$ Notice that there is a choice when performing this step, in case both the premisses of the cut-node are premisses of an $a x$-node: clearly, the two possibilities yield the same graph after reduction.
}

\section{Proposition 3 (Preservation of correction)}

If $\mathcal{R}$ is a net and $\mathcal{R}$ reduces to $\mathcal{R}^{\prime}$, then $\mathcal{R}^{\prime}$ is a net.

Proof. $\mathcal{R}^{\prime}$ is clearly a proof-structure. The fact that, moreover, $\mathcal{R}^{\prime}$ is a net is standard (see [9]).

We said that we are extending the Danos-Regnier's notion of untyped proof-nets. Indeed, a Danos-Regnier's untyped proof-net (see [9] for the precise definition) is a net: the edges labelled by ?I become ?-labelled and the other labels are erased.

Notice that cut-elimination does not hold for nets, for two different reasons:

- Deadlocks: normal nets might contain cuts. Take two cut-free nets and connect them by means of a cut-node whose premisses are not dual. Such a graph is a normal net which is not cut-free: the cut-node cannot be eliminated according to definition 8.

- The calculus is untyped: some nets have no normal form. Danos-Regnier's untyped proof-nets were able to encode the untyped $\lambda$-calculus. This is still the case for our nets, which means that the cut-elimination procedure applied to the nets corresponding to the fixpoints of the untyped $\lambda$-calculus never leads to a normal net.

\section{Proposition 4 (Confluence)}

If a net $\mathcal{R}$ reduces to $\mathcal{R}_{1}$ and $\mathcal{R}_{2}$ by some sequences of reductions, then there exists a net $\mathcal{R}^{\prime}$ such that both $\mathcal{R}_{1}$ and $\mathcal{R}_{2}$ can be reduced to $\mathcal{R}^{\prime}$.

\section{Remark 1}

Proof-nets were introduced in [13]. For additive-free second order LL, the notion currently used (and that we consider here) is obtained by combining [13, 9, 14], like in [24].

With every proof-net is naturally associated a net: simply forget formulas and erase the quantifier nodes. Notice that such a net is always deadlock-free. Conversely, it is sometimes possible to label a (deadlock-free) net by means of $\mathrm{LL}$ formulas (in such a way that the type of a ?-labelled edge is of the shape ?A) thus obtaining a proof-net: when this is the case we say that the net is typable.

With every sequent calculus proof (of additive-free second order LL) is associated a proof-net, and thus a net.

\section{Proposition 5 (Simulation)}

If $\mathcal{R}$ is a proof-net, let us denote by $\mathcal{R}^{-}$the net associated with $\mathcal{R}$. If $\mathcal{R}^{-}$reduces to $\mathcal{S}^{-}$in one reduction step then $\mathcal{R}$ reduces to $\mathcal{S}$ in at least one reduction step.

Proof. This is because cut-elimination for proof-nets does not depend on the formulas labelling the edges of the proofnets (only the nodes matter). One might have more than one step from $\mathcal{R}$ to $\mathcal{S}$ because of the presence of second order nodes in proof-nets (which are not present in the corresponding nets). 


\section{Proposition 6}

- The reduct of any typable net is typable (thus deadlockfree).

- Every typable net is strongly normalizing.

- The normal form of a typable net is unique and cut-free.

\section{Untyped ELL and SLL}

We are going to define restrictions of nets corresponding to (untyped versions of) the systems ELL [15] and SLL [17].

\section{Definition 9 (Exponential tree)}

An exponential tree is a tree whose nodes are: binary ?cnodes, unary ? $p$-nodes, 0 -ary $? w$-nodes and 0 -ary $? d$-nodes.

Let $a$ be a ?-labelled edge of the net $\mathcal{R}$, its exponential tree is given according to the node it is conclusion of:

- for a ? $d$-node, the exponential tree of $a$ is ?

- for a ?w-node, the exponential tree of $a$ is $2 w$

- for a ? $p$-node whose premisse is $a^{\prime}$, the exponential tree of $a$ is obtained by adding a ? $p$-node under the root of the exponential tree of $a^{\prime}$ :

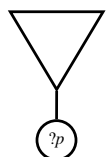

- for a ?c-node whose premisses are $a^{\prime}$ and $a^{\prime \prime}$, the exponential tree of $a$ is obtained by adding a ?c-node under the roots of the exponential trees of $a^{\prime}$ and $a^{\prime \prime}$ :

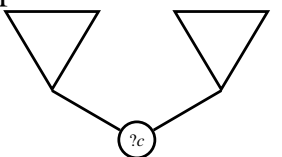

An exponential tree of $\mathcal{R}$ is maximal if it is the exponential tree of a ?-labelled edge which is not a premisse of a ? $p$ or ?c-node in $\mathcal{R}$.

\section{Definition 10 (Exponential bundle of branches)}

An exponential bundle of branches is a multiset of nonempty total orders (i.e. "filiform" trees or branches).

The exponential bundle of branches associated with an exponential tree is obtained in the following way:

- if the root of the tree is a ?d-node, then (the tree is reduced to this ? $d$-node and) the bundle is reduced to one branch which is itself reduced to one node

- if the root of the tree is a ?p-node under a tree $\mathcal{T}$, we consider the bundle $\mathcal{F}$ associated with $\mathcal{T}$ and we add a node under each branch

- if the root of the tree is a ?w-node, then (the tree is reduced to this ?w-node and) the associated bundle is empty

- if the root of the tree is a ?c-node under the trees $\mathcal{T}_{1}$ and $\mathcal{T}_{2}$, and if the associated bundles are $\mathcal{F}_{1}$ and $\mathcal{F}_{2}$, we obtain the (multiset) union of $\mathcal{F}_{1}$ and $\mathcal{F}_{2}$.

This mainly corresponds to extracting the multiset of branches ending with ?d-nodes in the tree.
The exponential bundle of branches of an edge of a net is the exponential bundle of branches associated with its exponential tree. The exponential bundles of branches of a net are the exponential bundles associated with its maximal exponential trees (see figure 4 for an example).

\section{Definition 11 (Untyped ELL and SLL)}

The systems $u E L L$ and $u S L L$ are defined as restrictions on nets:

- a net is in $u$ ELL if its exponential bundles of branches are all of the shape:

that is with only branches of length 2 .

- a net is in $u S L L$ if its exponential bundles of branches are all of one of the following two shapes:

that is either with only branches of length 1 or the bundle containing exactly one branch of length 2 .

These constraints on nets are not preserved by arbitrary reduction, but it is possible to define a strategy which preserves them: each time we reduce a cut with a ?-labelled premisse, we reduce the newly created cuts until the exponential tree of the original ?-labelled edge has been completely destroyed. In particular, if $\mathcal{R} \in u$ ELL (resp. $\mathcal{R} \in$ $u \mathrm{SLL}$ ) then its normal form is in $u \mathrm{ELL}$ (resp. $u \mathrm{SLL}$ ). See appendix A for more details.

\section{Proposition 7 (Complexity of representable functions)}

- $f$ is an elementary time function from integers to integers iff it is representable in $u \mathrm{ELL}$.

- $P$ is a polynomial time predicate over binary integers iff it is representable in $u \mathrm{SLL}$.

Proof. See appendix B.

\section{Semantics}

By adapting the notion of obsessional clique of section 2.2 to the untyped syntactical setting we have developed in the previous section, it is now possible to define models of $u \mathrm{ELL}$ and $u \mathrm{SLL}$.

\subsection{A space for untyped obsessionality}

We define points by the following grammar:

$x::=1|\perp| x \otimes x|x \not \gamma x| ! \mu \mid ? \mu \quad \mu::=\left[x_{1}, \ldots, x_{n}\right]$ where $\left[x_{1}, \ldots, x_{n}\right]$ denotes a finite multiset of points. $D$ is the set of all points ${ }^{4}$, and ? $D$ is the subset of $D$ containing only elements of the shape ? $\mu$. A clique is a subset of $D$.

\footnotetext{
${ }^{4}$ We can also define $D_{0}=\{1, \perp\}, D_{n+1}=D_{n} \cup\{(\otimes, x, y) \mid$ $\left.(x, y) \in D_{n}^{2}\right\} \cup\left\{(\gamma, x, y) \mid(x, y) \in D_{n}^{2}\right\} \cup\left\{(!, \mu) \mid \mu \in \mathcal{M}_{f}\left(D_{n}\right)\right\} \cup$ $\left\{(?, \mu) \mid \mu \in \mathcal{M}_{f}\left(D_{n}\right)\right\}$, and $D=\cup_{n=0}^{\infty} D_{n}$.
} 
We define the dual $\bar{x}$ of the point $x$ by $\overline{1}=\perp, \bar{\perp}=$ $1, \overline{x \otimes y}=\bar{x}$ × $\bar{y}, \overline{x^{2 \gamma y}}=\bar{x} \otimes \bar{y}, \overline{!\left[x_{1}, \ldots, x_{n}\right]}=$ $?\left[\overline{x_{1}}, \ldots, \overline{x_{n}}\right]$ and $\overline{?\left[x_{1}, \ldots, x_{n}\right]}=!\left[\overline{x_{1}}, \ldots, \overline{x_{n}}\right]$.

\section{Definition 12 (Experiment)}

An experiment of a net $\mathcal{R}$ is defined by induction on the depth of $\mathcal{R}$. It is a labelling of the 0 -depth edges with elements of $D$ such that:

- if the edge is ?-labelled, the label is an element of ?D

- if $x$ is the label of a conclusion of an $a x$-node, the label of the other one is $\bar{x}$

- if $x$ is the label of a premisse of a cut-node, the label of the other one is $\bar{x}$

- if $x$ is the label of the conclusion of a 1-node (resp. $\perp$-node) then $x=1$ (resp. $x=\perp$ )

- if $x$ and $y$ are the labels of the premisses of a $\otimes$-node (resp. 2 -node) then the label of the conclusion is $x \otimes y$ (resp. $x>8$ )

- if $x$ is the label of the premisse of a ?d-node then the label of the conclusion is ? [x]

- if $x$ is the label of the conclusion of a ?w-node then $x=$ ?[]

- if $x$ and $y$ are the labels of the premisses of a ?c-node, they have the shape $x=? \mu$ and $y=? \nu$, and the label of the conclusion must be ? $(\mu+\nu)$

- if $x$ is the label of the conclusion of a !-node and $x_{1}, \ldots, x_{n}$ are the labels of the conclusions of the auxiliary doors of the corresponding box then we must have $x=!\left[y_{1}, \ldots, y_{k}\right]$ and there must exist $k$ experiments of the content of the box associating the labels $y_{i}, ? \mu_{1}^{i}, \ldots, ? \mu_{n}^{i}$ with the conclusions of the content of the box with the property $x_{j}=?\left(\mu_{j}^{1}+\cdots+\mu_{j}^{k}\right)$ for $1 \leq j \leq n$.

The result of an experiment is the point $\left(\cdots\left(\left(x_{1} \not \gamma x_{2}\right)\right.\right.$ > $\left.x_{3}\right) \ldots$ ) $2 x_{n}$ where $x_{1}, \ldots, x_{n}$ are the labels of the conclusions of the net. The semantics $\llbracket \mathcal{R} \rrbracket$ of a net $\mathcal{R}$ is the set of the results of its experiments, thus a clique.

An $h$-experiment is an experiment which takes exactly $h$ experiments in the content of each box.

\section{Theorem 3 (Correctness)}

If $\mathcal{R}$ reduces to $\mathcal{R}^{\prime}$ then $\llbracket \mathcal{R} \rrbracket=\llbracket \mathcal{R}^{\prime} \rrbracket$.

Proof. See [13] for coherent semantics, the relational case we consider here is almost the same.

\section{Remark 2}

A 1-experiment is obtained by putting exactly one label on each conclusion of each $a x$-node of a net (in fact a pair of dual labels for the pairs of conclusions of the $a x$-nodes), and by propagating labels in a top-down way (in particular when crossing a box, we just propagate labels with $x \mapsto ![x]$ for the main door). This is always possible for a cut-free net and thus experiments of cut-free nets always exist. This entails that the semantics of a net having a cut-free normal form is never empty.

\section{Definition 13 (Action)}

Let $t \geq 0$ be a natural number, the $t$-action on $D$ is the function $(k, x) \mapsto(x)_{t}^{(k)}$ from $\mathbb{N}^{*} \times D$ to $D$ given by:

$$
\begin{gathered}
(1)_{t}^{(k)}=1 \quad(\perp)_{t}^{(k)}=\perp \\
(x \otimes y)_{t}^{(k)}=(x)_{t}^{(k)} \otimes(y)_{t}^{(k)} \\
(x \ngtr y)_{t}^{(k)}=(x)_{t}^{(k)} \ngtr(y)_{t}^{(k)} \\
\left(!\left[x_{1}, \ldots, x_{n}\right]\right)_{t}^{(k)}= \begin{cases}!\left[\left(x_{1}\right)_{t}^{(k)}, \ldots,\left(x_{n}\right)_{t}^{(k)}\right] & \text { if } n \leq t \\
!\left[k\left(x_{1}\right)_{t}^{(k)}, \ldots, k\left(x_{n}\right)_{t}^{(k)}\right] & \text { if } n>t\end{cases} \\
\left(?\left[x_{1}, \ldots, x_{n}\right]\right)_{t}^{(k)}= \begin{cases}?\left[\left(x_{1}\right)_{t}^{(k)}, \ldots,\left(x_{n}\right)_{t}^{(k)}\right] & \text { if } n \leq t \\
?\left[k\left(x_{1}\right)_{t}^{(k)}, \ldots, k\left(x_{n}\right)_{t}^{(k)}\right] & \text { if } n>t\end{cases}
\end{gathered}
$$

A clique $c$ is t-obsessional if $\forall x \in c, \forall k \in$ $\mathbb{N}^{*},(x)_{t}^{(k)} \in c$. A clique $c$ is obsessional from $t$ if for any $t^{\prime} \geq t, c$ is $t^{\prime}$-obsessional.

\subsection{Models of $u \mathrm{ELL}$ and $u \mathrm{SLL}$}

If we define $D_{i}$ as $D$ equipped with the $i$-action, then $D_{0}$ and $\left(D_{i}\right)_{i \in \mathbb{N}}$ are reflexive objects respectively in $\mathcal{O R E} \mathcal{L}$ and $\mathcal{S R \mathcal { E } L}$. Meaning that $1, \perp, D_{0} \otimes D_{0}, D_{0}$ > $D_{0}, ! D_{0}$ and ? $D_{0}$ are retracts of $D_{0}$ in $\mathcal{O} \mathcal{R} \mathcal{E} \mathcal{L}$ (and the same for $\left(D_{i}\right)_{i \in \mathbb{N}}$ in $\mathcal{S R E} \mathcal{L}$ ).

\section{Proposition 8 (Models)}

- 0-obsessional cliques in $D$ are a model of $u \mathrm{ELL}$.

- Cliques which are obsessional from some t are a model of $u \mathrm{SLL}$.

\subsection{Relative completeness}

We are going to prove a converse of this last proposition. While every proof in $u \mathrm{ELL} / u \mathrm{SLL}$ is interpreted by an obsessional clique (0-obsessional for $u$ ELL and obsessional from some $t$ for $u S L L)$, it is clear that there exist obsessional cliques which are not the interpretation of a proof of the corresponding system ( $u$ ELL or $u S L L)$ mainly because the relational model is not complete for LL (and even not for MLL which is included in both $u E L L$ and $u S L L$ and for which any clique is obsessional). Nevertheless, we can ask this question for a clique for which we already know that it is the interpretation of an LL-proof: if this clique is obsessional, is it the interpretation of a $u \mathrm{ELL} / u$ SLL-proof? A positive answer is what we call relative completeness and it will be the main result of the paper: theorem 4 .

\section{Definition 14 ( $h$-point)}

For a given $h \in \mathbb{N}$, h-points are the elements of $D$ inductively given by: 
- 1 and $\perp$ are $h$-points

- $x \otimes y$ and $x$ ×8 $y$ are $h$-points if $x$ and $y$ are $h$-points

- ? $\left[x_{1}, \ldots, x_{n}\right]$ is an $h$-point if $x_{1}, \ldots, x_{n}$ are $h$-points

- ! $\left[x_{1}, \ldots, x_{n}\right]$ is an $h$-point if $n=h$ and $x_{1}, \ldots, x_{n}$ are $h$-points

\section{Lemma 1 ( $h$-points and $h$-experiments)}

Let $\mathcal{R}$ be a cut-free net and $x$ be the result of an experiment $e$ of $\mathcal{R}, x$ is an h-point iff $e$ is an h-experiment.

Proof. See appendix C.

\section{Definition 15 (?-trees)}

Let $x$ be an element of $D, \mathcal{T}(x)$ is the subset of $\mathbb{N}$ defined inductively by:

- $\mathcal{T}(1)=\mathcal{T}(\perp)=\emptyset$

- $\mathcal{T}(x \otimes y)=\mathcal{T}(x>8 y)=\mathcal{T}(x) \cup \mathcal{T}(y)$

- $\mathcal{T}\left(!\left[x_{1}, \ldots, x_{n}\right]\right)=\mathcal{T}\left(x_{1}\right) \cup \cdots \cup \mathcal{T}\left(x_{n}\right)$

- $\mathcal{T}\left(?\left[x_{1}, \ldots, x_{n}\right]\right)=\{n\} \cup \mathcal{T}\left(x_{1}\right) \cup \cdots \cup \mathcal{T}\left(x_{n}\right)$

\section{Lemma 2 (Action on ?-trees)}

Let $x$ be an element of $D, h>0$ and $t \geq 0$ be two natural numbers, we have:

$$
\begin{aligned}
& \mathcal{T}\left((x)_{t}^{(h)}\right)= \\
& \{h n \mid n \in \mathcal{T}(x) \wedge n>t\} \cup\{n \mid n \in \mathcal{T}(x) \wedge n \leq t\}
\end{aligned}
$$

Proof. See appendix D.

With the base $h$ representation of a number $n$ (denoted by $\bar{n}^{h}$ ), it is possible to associate an exponential bundle of branches. If $\bar{n}^{h}=n_{k} \ldots n_{0}$, we consider the bundle containing $n_{0}+\cdots+n_{k}$ branches and: for each $0 \leq i \leq k$, we have $n_{i}$ branches of length $i+1$. For example, $\overline{11}^{3}=102$ and the corresponding exponential bundle is:

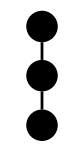

The contraction size $\operatorname{cosize}(\mathcal{R})$ of a net $\mathcal{R}$ is the maximum number of branches of its exponential bundles of branches. In particular, if $\mathcal{R}$ is a net associated with a sequent calculus proof $\pi$ in SLL, its contraction size is the maximal width of the ? $m$-rules of $\pi$.

\section{Lemma 3 (Computation of bundles of branches)}

Let $\mathcal{R}$ be a cut-free net, if $h>\operatorname{cosize}(\mathcal{R})$ and if $x$ is an $h$-point of $\llbracket \mathcal{R} \rrbracket$ then $\mathcal{T}(x)$ is the set of all $n$ such that $\bar{n}^{h}$ is an exponential bundle of branches of $\mathcal{R}$.

Proof. Using $h>\operatorname{cosize}(\mathcal{R})$, we first show that, in any $h$-experiment, the size of the multiset associated with a ?labelled edge is $n$ where $\bar{n}^{h}$ corresponds to the exponential bundle of branches of this edge.

We can now verify, by induction on $\mathcal{R}$, that if $x$ is the result of an experiment $e$ of $\mathcal{R}$ then $\mathcal{T}(x)$ contains exactly the sizes of the labels associated (by some experiment used to build $e$, either at depth 0 or inside a box) with the ?labelled edges of $\mathcal{R}$ which are not premisse of a $? p$ or $? c$ node. We conclude with lemma 1.

See appendix E for more details.

We have already seen (remark 2) how 1-experiments are built. It is immediate that the result $x$ of such an experiment is a 1 -point with $\forall n \in \mathcal{T}(x), n \leq \operatorname{cosize}(\mathcal{R})$.

More generally, starting from a 1-experiment, we can repeat it $h$ times in each box. This is always possible for a cut-free net and gives an $h$-experiment thus an $h$-point.

\section{Theorem 4 (Relative completeness) \\ Let $\mathcal{R}$ be a cut-free net,

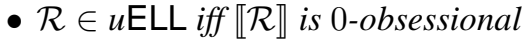 \\ - $\mathcal{R} \in u \mathrm{SLL}$ iff $\llbracket \mathcal{R} \rrbracket$ is obsessional from some $t$}

Proof. We have already seen (see proposition 8) that if $\mathcal{R} \in$ $u \mathrm{ELL}$ then $\llbracket \mathcal{R} \rrbracket$ is 0 -obsessional.

Let $x$ be the result of a 1-experiment of $\mathcal{R}$ and let $h$ be a natural number such that $h>\operatorname{cosize}(\mathcal{R})$. If $\llbracket \mathcal{R} \rrbracket$ is 0 obsessional, one has $(x)_{0}^{(h)} \in \llbracket \mathcal{R} \rrbracket$ which is an $h$-point. If $\mathcal{R}$ is not in $u$ ELL, then it contains an exponential bundle of branches such that:

- a branch contains at least three nodes: by lemma 3 there exists $n \in \mathcal{T}\left((x)_{0}^{(h)}\right)$ such that $\bar{n}^{h}=n_{k} \ldots n_{0}$ with $n_{j} \geq 1(j \geq 2)$ thus $n \geq h^{2}$. This is impossible since, by lemma 2 , the elements of $\mathcal{T}\left((x)_{0}^{(h)}\right)$ are bounded by $h \cdot \operatorname{cosize}(\mathcal{R})<h^{2}$.

- a branch has length 1: by lemma 3 there exists $n \in$ $\mathcal{T}\left((x)_{0}^{(h)}\right)$ such that $\bar{n}^{h}=n_{k} \ldots n_{0}$ with $n_{0} \geq 1$. This is impossible, by lemma 2 , since the sizes of the multisets of $(x)_{0}^{(h)}$ are all multiples of $h$.

We have already seen (see proposition 8) that if $\mathcal{R} \in$ $u \mathrm{SLL}$ then $\llbracket \mathcal{R} \rrbracket$ is obsessional from some $t$.

Conversely, there exists some $t>\operatorname{cosize}(\mathcal{R})$ such that $\llbracket \mathcal{R} \rrbracket$ is $t$-obsessional. Let $x$ be the $t$-point obtained by repeating $t$ times in each box a 1-experiment of $\mathcal{R}$. By lemma 3 , the elements of $\bigcup_{x t \text {-point } \in \llbracket \mathcal{R} \rrbracket} \mathcal{T}(x)$ are bounded. Since $x$ is a $t$-point, we can easily check that $(x)_{t}^{(h)}$ is also a $t$-point (for any $h \in \mathbb{N}^{*}$ ). If we consider the family of $t$-points $\left((x)_{t}^{(h)}\right)_{h \in \mathbb{N}^{*}}$ of $\llbracket \mathcal{R} \rrbracket$, boundedness entails by lemma 2 that $\forall n \in \mathcal{T}(x), n \leq t$. By lemma 3, any exponential bundle of branches in $\mathcal{R}$ corresponds to a natural number $n$ (with $\bar{n}^{t}=n_{k} \ldots n_{0}$ ) belonging to $\mathcal{T}(x)$. Since $n \leq t$, we have either $k=1, n_{1}=1$ and $n_{0}=0$, or $k=0$. So that $\mathcal{R}$ is in $u$ SLL.

The restriction to cut-free nets is perfectly reasonable since the encoding of elementary/polynomial time algorithms is done with typable nets. As shown in proposition 6, the reduction of such nets leads to cut-free normal forms 
(and by correctness of the model, the interpretation of a net and of its normal form are the same).

\section{Conclusion}

Let us first comment on different choices we made:

- The multiplicative-exponential setting is powerful enough to express elementary/polynomial time in our untyped context. However it would be possible to add the additive connectives in order to simplify the encoding of certain computations. In the case of ELL, this can be done without any problem (in the spirit of [10]) and leads to an extension of the relative completeness result. In the case of SLL, things are much more complicated and the interaction between additive and exponential connectives is not clear enough (up to now) to get relative completeness.

- The model we consider is based on the relational model of LL. The use of "multiset based" coherent semantics would change nothing except that we would have to define the various constructions applied to the coherence relation. Our choice of the relational setting is only justified by the induced simplicity of the presentation.

- Concerning subsystems of LL for polynomial time, instead of SLL, a very natural choice would be LLL [15]. However we would face two different problems. First, relative completeness (as stated here) for LLL (as given in [15]) would fail for coherent semantics. The second problem is that our approach by obsessionality gives an analysis of proof-nets according to their exponential trees without any consideration about the different boxes crossed by these trees. In LLL, the "at most one auxiliary door" constraint would require to compare the boxes with which the ?p-nodes are associated and this is something we are not able to do at the moment.

Concerning the other extensions of our work, the most natural one is to try to find relative completeness results with other models, other subsystems of LL (trying to say something for LLL for example), ... Moreover many systems for bounded time complexity are defined as subsystems of the $\lambda$-calculus and it is natural to address the question of relative completeness in the setting of the $\lambda$-calculus instead of LL.

Another direction is related to the main defect of relative completeness: it tells us something about the obsessional elements of the model which are interpretations of proofs of LL but nothing for the other obsessional elements. What would be very nice, in the context of implicit computational complexity, is to find a property which is both "absolute for complexity" (meaning that any element of the model satisfying the property would be in a given complexity class, this element being the interpretation of a proof or not) and relatively complete.
Acknowledgments. We would like to thank P. Baillot who has been our reference for various informations about ELL, LLL, SLL, ... and who suggested us to use an untyped setting for encoding second order. We also thank P. Boudes, T. Ehrhard, D. Mazza and P.-A. Melliès for discussions related to the topics of this paper.

\section{References}

[1] A. Asperti. Light affine logic. In LICS. IEEE Computer Society Press, 1998.

[2] A. Asperti and L. Roversi. Intuitionistic light affine logic. ACM Trans. on Computational Logic, 1(3):1-39, 2002.

[3] P. Baillot. Stratified coherent spaces: a denotational semantics for light linear logic. Theoretical Computer Science, 318(1-2):29-55, 2004.

[4] S. J. Bellantoni and S. A. Cook. A new recursion-theoretic characterization of the polytime functions. Computational Complexity, 2:97-110, 1992.

[5] S. R. Buss. Bounded Arithmetic. Ph.D. thesis, Princeton University, 1985.

[6] A. Cobham. The intrinsic computational difficulty of functions. In International Congress for Logic, Methodology, and the Philosophy of Science. North-Holland, 1964.

[7] S. Cook. The complexity of theorem proving procedures. In Conference Record of Third Annual ACM Symposium on Theory of Computing, pages 151-158, 1971.

[8] U. Dal Lago and M. Hofmann. Quantitative models and implicit complexity. In Proceedings of FSTTCS, volume 3821 of LNCS. Springer, 2005.

[9] V. Danos. La Logique Linéaire appliquée à l'étude de divers processus de normalisation (principalement $d u \lambda$-calcul). $\mathrm{Ph} . \mathrm{D}$. thesis, Université Paris VII, 1990.

[10] V. Danos and J.-B. Joinet. Linear logic and elementary time. Information and Computation, 183(1):123-137, 2003.

[11] D. de Carvalho. Ph.D. thesis, Université Aix-Marseille II. In preparation.

[12] R. Fagin. Generalized first-order spectra and polynomialtime recognizable sets. In Complexity and computation, SIAM-AMS Proceedings, pages 43-73, 1974.

[13] J.-Y. Girard. Linear logic. Theoretical Computer Science, 50:1-102, 1987.

[14] J.-Y. Girard. Quantifiers in linear logic II. In Nuovi problemi della logica e della filosofia della scienza. CLUEB, 1991.

[15] J.-Y. Girard. Light linear logic. Information and Computation, 143(2):175-204, 1998.

[16] J.-Y. Girard, A. Scedrov, and P. J. Scott. Bounded linear logic: a modular approach to polynomial time computability. Theoretical Computer Science, 97:1-66, 1992.

[17] Y. Lafont. Soft linear logic and polynomial time. Theoretical Computer Science, 318(1-2):163-180, 2004.

[18] D. Leivant and J.-Y. Marion. Lambda calculus characterizations of poly-time. Fundamenta Informaticae, 19(1-2):167184, 1993.

[19] H. Mairson and K. Terui. On the computational complexity of cut-elimination in linear logic. In Proceedings of ICTCS, volume 2841 of $L N C S$, pages 23-36. Springer, 2003. 
[20] D. Mazza. Logica Lineare e Complessità Computazionale. Tesi di laurea, Università di Roma Tre, 2002.

[21] A. Murawski and C.-H. L. Ong. Discreet games, light affine logic and ptime computation. In CSL, volume 1862 of LNCS, pages 55-92. Springer, 2000.

[22] B. Redmond. Multiplexor categories and models of soft linear logic. Ph.D. thesis, University of Ottawa. In preparation.

[23] L. Regnier. Lambda-Calcul et Réseaux. Ph.D. thesis, Université Paris VII, 1992.

[24] L. Tortora de Falco. Réseaux, cohérence et expériences obsessionnelles. Ph.D. thesis, Université Paris VII, 2000.

[25] L. Tortora de Falco. Obsessional experiments for linear logic proof-nets. Mathematical Structures in Computer Science, 13(6):799-855, 2003.

\section{Appendix}

\section{A. Preservation of $u E L L$ and $u S L L$ by reduc- tion}

An exponential cut is a cut between a ?-labelled edge and the conclusion of a !-node. Every exponential cut $c$ comes with a box: the one associated with the !-node whose conclusion is a premisse of the cut, to which we refer as the c-box.

If $c$ is an exponential cut in $\mathcal{R}$ and $\mathcal{R}^{\prime}$ is obtained by reducing $c$ in $\mathcal{R}$, the one-step residues of $c$ in $\mathcal{R}^{\prime}$ (which are also exponential cuts) are given by:

- if $c$ is a ?d cut, it has no one-step residue,

- if $c$ is a ? $w$ cut, it has no one-step residue,

- if $c$ is a ?p cut, its unique one-step residue is the new cut obtained from $c$

- if $c$ is a ?c cut, its one-step residues are the two new cuts obtained from $c$.

The residues of $c$ are given by: a one-step residue is a residue and a one-step residue of a residue is a residue.

A sequence of reductions focalizes on an exponential cut $c$ if it starts by reducing $c$ and then only reduces residues of $c$. A complete reduction of an exponential cut $c$ is a sequence of reductions which focalizes on $c$ and leads to a net without any residue of $c$ (thus it is a maximal sequence of reductions focalizing on $c$ ).

An edge a dominates an exponential cut $c$ if one of the auxiliary doors of the $c$-box is in the exponential tree of $a$.

If $c$ is an exponential cut, $a$ dominates $c$ and $\mathcal{T}$ is the exponential tree of the ?-labelled premisse of $c$, grafting $c$ on $a$ means: if $\mathcal{T}_{1}$ is the exponential tree of $a$, for every auxiliary door $n$ of the $c$-box belonging to $\mathcal{T}_{1}$, we consider the exponential tree $\mathcal{T}_{2}$ of the premisse of $n$, we replace the ? $d$-nodes of $\mathcal{T}$ by copies of $\mathcal{T}_{2}$ and we graft the resulting tree instead of $n$ (and $\mathcal{T}_{2}$ ) in $\mathcal{T}_{1}$.

We consider a net $\mathcal{R}$, an exponential cut $c$ in $\mathcal{R}$, an edge $a$ which is a root of a maximal exponential tree and which dominates $c$ in $\mathcal{R}$, and a net $\mathcal{R}^{\prime}$ such that $\mathcal{R}$ reduces to $\mathcal{R}^{\prime}$ by a sequence of reductions focalizing on $c$. If we still call $a$ the unique edge of $\mathcal{R}^{\prime}$ corresponding to $a$, the tree obtained by grafting $c$ on $a$ in $\mathcal{R}$ is the same as the tree obtained by grafting all the residues of $c$ on $a$ in $\mathcal{R}^{\prime}$. We show it in the particular case where $a$ is the conclusion of an auxiliary door of the $c$-box. By induction on the length of the reduction from $\mathcal{R}$ to $\mathcal{R}^{\prime}$, we look at each possible reduction step (using the notations $\mathcal{T}, \mathcal{T}_{1}$ and $\mathcal{T}_{2}$ as given above, the assumption on $a$ corresponding to $\mathcal{T}_{1}$ obtained from $\mathcal{T}_{2}$ by adding a ?p-node):

- ? $d$-step: before reduction $\mathcal{T}$ is a ? $d$-node and the result of grafting is thus $\mathcal{T}_{2}$, while after reduction we directly have $\mathcal{T}_{2}$ (there is no grafting); 
- ?w-step: before reduction $\mathcal{T}$ is a ?w-node and the result of grafting is thus just a ?w-node, while after reduction we directly have a ?w-node (there is no grafting);

- ?p-step: before reduction $\mathcal{T}$ is a ?p-node under a tree $\mathcal{T}^{\prime}$ and the graft gives $\mathcal{T}$ with ? $d$-nodes replaced by copies of $\mathcal{T}_{2}$, while after reduction the graft gives a ?p-node under $\mathcal{T}^{\prime}$ with ? $d$-nodes replaced by copies of $\mathcal{T}_{2}$ (that is the same as before reduction);

- ?c-step: before reduction $\mathcal{T}$ is a ?c-node under two trees $\mathcal{T}^{\prime}$ and $\mathcal{T}^{\prime \prime}$ and the graft gives $\mathcal{T}$ with ? $d$-nodes replaced by copies of $\mathcal{T}_{2}$, while after reduction the graft gives a ?c-node under $\mathcal{T}^{\prime}$ with ? $d$-nodes replaced by copies of $\mathcal{T}_{2}$ and $\mathcal{T}^{\prime \prime}$ with ? $d$-nodes replaced by copies of $\mathcal{T}_{2}$ (that is the same as before reduction).

Let $c$ be an exponential cut in $\mathcal{R}$ and let $a$ be an edge which dominates $c$ and which is the root of a maximal exponential tree, we consider a complete reduction of $c$ from $\mathcal{R}$ to some $\mathcal{R}^{\prime}$. By the result above, the exponential tree of $a$ in $\mathcal{R}^{\prime}$ is the same as the one obtained by grafting $c$ on $a$ in $\mathcal{R}$. We now show that grafting an exponential cut on the root of a maximal exponential tree preserves the fact of being in $u E L L$ and the same for $u S L L$ :

- In $u$ ELL, during a graft, we replace ? $d$-nodes in $\mathcal{T}$ (which contains exactly one ?p-node on the branches leading to these ? $d$-nodes) by copies of $\mathcal{T}_{2}$ (which cannot contain a ?p-node on a branch leading to a $? d$ leaf) and we put the result in place of a subtree with root a ? $p$-node in $\mathcal{T}_{1}$ (which contains no other ?p-nodes on a branch leading to a ? $d$-node), in this way we get an $u E L L$ valid exponential bundle of branches.

- In $u$ SLL, we are necessarily grafting on a tree $\mathcal{T}_{1}$ which gives an exponential bundle of branches restricted to one branch of length 2 . It is easy to check that grafting $\mathcal{T}$ on such a tree leads to the same bundle as for $\mathcal{T}$.

Moreover, if $\mathcal{T}_{1}$ is the tree reduced to one ?d-node and one ? $p$-node, then grafting $\mathcal{T}$ on $\mathcal{T}_{1}$ leads to $\mathcal{T}$ (thus new trees are never created during complete reduction in this particular case).

\section{B. Proof of proposition 7}

The size of a net $\mathcal{R}$ is its number of nodes.

For completeness, we just apply the results of section 2.1, and we translate the proofs representing functions and predicates into nets.

For the elementary time correctness for $u \mathrm{ELL}$, we consider a particular reduction strategy:

a. we reduce all the multiplicative (i.e. non-exponential) cuts

b. we apply complete reductions to the exponential cuts (in order to stay in $u$ ELL)

based on the fact that the reduction of an exponential cut in $u E L L$ cannot create new multiplicative cuts at its own depth.
In a net without any multiplicative cut, we can define a notion of ?-path: a ?-path is a path starting from a !-node, going downward from one of the auxiliary doors of its box through only?-labelled edges until it reaches an exponential (non deadlock) cut then it goes to the !-node premisse of the cut, and so on... These paths stop in deadlock cuts, in ? edges which are not premisse of a node with a ? conclusion and in conclusions of the net.

An exponential cut is maximal if there is no ?-path from it going from its! premisse to another exponential cut (such maximal cuts always exist by correctness of nets). If we restrict our complete reductions of exponential cuts to maximal exponential cuts, we can check that such a reduction never modifies the exponential tree of another exponential cut.

We remark that the reduction of a multiplicative cut decreases the number of nodes of the net and that the complete reduction of a maximal exponential cut at depth $k$ decreases the number of nodes at depth $k$. We can deduce that the full reduction of a net at depth $k$ can be done in a number of steps bounded by the square of the size of the net.

Finally, since each reduction step can at most double the size of the net, we conclude that the reduction of a net can be done in a number of steps bounded by a tower of exponentials applied to the size of the original net and whose height depends linearly on the depth of the original net (since the (complete) reduction steps can only decrease this depth).

The representation of the integer $n$ is given by a net of size linear in $n$ and depth 1 . If $f$ is represented by a net of depth $d$ then the normalization of $f$ cut with $n$ is thus done in elementary time with respect to $n$.

For the polynomial time correctness for $u \mathrm{SLL}$, we first associate a polynomial $W_{\mathcal{R}}(X)$ to any net $\mathcal{R}$ of $u \mathrm{SLL}$ :

- if it contains no box, then $W_{\mathcal{R}}(X)$ is its number of $a x$, $\otimes, \not \gamma, 1, \perp$ and ?d-nodes,

- if it contains boxes $B_{1}, \ldots, B_{k}$ containing the nets $\mathcal{R}_{1}, \ldots, \mathcal{R}_{k}$, and $N$ is the number of $a x, \otimes, \mathcal{X}, 1, \perp$ and ? $d$-nodes at depth 0 , then $W_{\mathcal{R}}(X)=N+X(1+$ $\left.W_{\mathcal{R}_{1}}(X)\right)+\cdots+X\left(1+W_{\mathcal{R}_{k}}(X)\right)$.

Assuming $n \geq 1$, any multiplicative reduction decreases $W_{\mathcal{R}}(n)$. Moreover if we consider a complete exponential reduction, we have two possible cases for the exponential bundle of branches:

- only one branch of length 2: the reduction replaces a term $X+X\left(1+W_{\mathcal{R}}(X)\right)$ by $X W_{\mathcal{R}}(X)$. Moreover this complete reduction takes as many steps as the size of the cut exponential tree;

- $p$ branches of length 1: the reduction replaces a term $p+X\left(1+W_{\mathcal{R}}(X)\right)$ by $p W_{\mathcal{R}}(X)$. Moreover this complete reduction takes as many steps as the size of the cut exponential tree;

so that if $n$ is bigger than the sizes of all the exponential trees occurring during the reduction of $\mathcal{R}$ then the length of 
the reduction of $\mathcal{R}$ is bounded by $W_{\mathcal{R}}(n)$.

The representation of a binary integer $b$ of length $n$ is given by a net of size linear in $n$, depth 0 and containing one exponential tree which is of size smaller than $2 n+1$. A predicate $P$ is represented by a net without ?c-node. As a consequence, the normalization of $P$ (having $k$ conclusions corresponding to $\mathbb{W}^{\perp}$ ) cut with $k$ copies of $b$ is done in at most $W_{P}(2 n+1)+k O(n)$ steps which is a polynomial in $n$. We use here the fact that during the normalization of the whole net, all the exponential trees will be copies (or subtrees) of the ones of $b$ as shown at the end of appendix A.

\section{Proof of lemma 1}

By induction on $\mathcal{R}$ :

- If $\mathcal{R}$ has a final $a x, 1, \perp, \otimes, \not \gamma, ? d, ? w$ or ?c-node, the result is immediate by induction hypothesis.

- If $\mathcal{R}$ has two connected components, we also apply the induction hypothesis to the components.

- If $\mathcal{R}$ is reduced to a box, $e$ is obtained from some experiments $e_{1}, \ldots, e_{n}$ in the box. If $x$ is an $h$-point, the size of the multiset associated with the conclusion of the !-node is $h$ thus $n=h$, and the results of $e_{1}, \ldots, e_{h}$ are $h$-points so that, by induction hypothesis, they are all $h$-experiments and finally $e$ is an $h$-experiment. If $e$ is an $h$-experiment, then the size of the multiset associated with the conclusion of the !-node is $h, n=h$ and $e_{1}, \ldots, e_{h}$ are $h$-experiments thus, by induction hypothesis, their results are $h$-points and finally $x$ is an $h$-point.

\section{Proof of lemma 2}

By induction on $x$ :

- $\mathcal{T}\left((1)_{t}^{(h)}\right)=\mathcal{T}(1)=\emptyset$ and the same for $\perp$

- by induction hypothesis:

$$
\begin{aligned}
& \mathcal{T}\left((x \otimes y)_{t}^{(h)}\right)= \mathcal{T}\left((x)_{t}^{(h)} \otimes(y)_{t}^{(h)}\right) \\
&= \mathcal{T}\left((x)_{t}^{(h)}\right) \cup \mathcal{T}\left((y)_{t}^{(h)}\right) \\
&=\{h n \mid n \in \mathcal{T}(x) \wedge n>t\} \\
& \cup\{n \mid n \in \mathcal{T}(x) \wedge n \leq t\} \\
& \cup\{h n \mid n \in \mathcal{T}(y) \wedge n>t\} \\
& \cup\{n \mid n \in \mathcal{T}(y) \wedge n \leq t\} \\
&=\{h n \mid n \in \mathcal{T}(x) \cup \mathcal{T}(y) \wedge n>t\} \\
& \cup\{n \mid n \in \mathcal{T}(x) \cup \mathcal{T}(y) \wedge n \leq t\} \\
&=\{h n \mid n \in \mathcal{T}(x \otimes y) \wedge n>t\} \\
& \cup\{n \mid n \in \mathcal{T}(x \otimes y) \wedge n \leq t\}
\end{aligned}
$$

- if $k \leq t$

$$
\begin{aligned}
& \mathcal{T}\left(\left(!\left[x_{1}, \ldots, x_{k}\right]\right)_{t}^{(h)}\right) \\
& =\mathcal{T}\left(!\left[\left(x_{1}\right)_{t}^{(h)}, \ldots,\left(x_{k}\right)_{t}^{(h)}\right]\right) \\
& =\mathcal{T}\left(\left(x_{1}\right)_{t}^{(h)}\right) \cup \cdots \cup \mathcal{T}\left(\left(x_{k}\right)_{t}^{(h)}\right) \\
& =\bigcup_{1 \leq i \leq k}\left\{h n \mid n \in \mathcal{T}\left(x_{i}\right) \wedge n>t\right\} \\
& \cup \bigcup_{1 \leq i \leq k}\left\{n \mid n \in \mathcal{T}\left(x_{i}\right) \wedge n \leq t\right\} \\
& =\left\{h n \mid n \in \bigcup_{1 \leq i \leq k} \mathcal{T}\left(x_{i}\right) \wedge n>t\right\} \\
& \cup\left\{n \mid n \in \bigcup_{1 \leq i \leq k} \mathcal{T}\left(x_{i}\right) \wedge n \leq t\right\} \\
& =\left\{h n \mid n \in \mathcal{T}\left(!\left[x_{1}, \ldots, x_{k}\right]\right) \wedge n>t\right\} \\
& \cup\left\{n \mid n \in \mathcal{T}\left(!\left[x_{1}, \ldots, x_{k}\right]\right) \wedge n \leq t\right\}
\end{aligned}
$$

- if $k>t$

$$
\begin{aligned}
\mathcal{T}\left(\left(!\left[x_{1}, \ldots, x_{k}\right]\right)_{t}^{(h)}\right) & =\mathcal{T}\left(!\left[h\left(x_{1}\right)_{t}^{(h)}, \ldots, h\left(x_{k}\right)_{t}^{(h)}\right]\right) \\
& =\mathcal{T}\left(\left(x_{1}\right)_{t}^{(h)}\right) \cup \cdots \cup \mathcal{T}\left(\left(x_{k}\right)_{t}^{(h)}\right)
\end{aligned}
$$

and we conclude in the same way

- if $k \leq t$

$$
\begin{aligned}
& \mathcal{T}\left(\left(?\left[x_{1}, \ldots, x_{k}\right]\right)_{t}^{(h)}\right) \\
& =\mathcal{T}\left(?\left[\left(x_{1}\right)_{t}^{(h)}, \ldots,\left(x_{k}\right)_{t}^{(h)}\right]\right) \\
& =\{k\} \cup \mathcal{T}\left(\left(x_{1}\right)_{t}^{(h)}\right) \cup \cdots \cup \mathcal{T}\left(\left(x_{k}\right)_{t}^{(h)}\right) \\
& =\{k\} \cup \bigcup_{1 \leq i \leq k}\left\{h n \mid n \in \mathcal{T}\left(x_{i}\right) \wedge n>t\right\} \\
& \cup \bigcup_{1 \leq i \leq k}\left\{n \mid n \in \mathcal{T}\left(x_{i}\right) \wedge n \leq t\right\} \\
& =\{k\} \cup\left\{h n \mid n \in \bigcup_{1 \leq i \leq k} \mathcal{T}\left(x_{i}\right) \wedge n>t\right\} \\
& \cup\left\{n \mid n \in \bigcup_{1 \leq i \leq k} \mathcal{T}\left(x_{i}\right) \wedge n \leq t\right\} \\
& =\left\{h n \mid n \in\left(\{k\} \cup \bigcup_{1 \leq i \leq k} \mathcal{T}\left(x_{i}\right)\right) \wedge n>t\right\} \\
& \cup\left\{n \mid n \in\left(\{k\} \cup \bigcup_{1 \leq i \leq k} \mathcal{T}\left(x_{i}\right)\right) \wedge n \leq t\right\} \\
& =\left\{h n \mid n \in \mathcal{T}\left(?\left[x_{1}, \ldots, x_{k}\right]\right) \wedge n>t\right\} \\
& \cup\left\{n \mid n \in \mathcal{T}\left(?\left[x_{1}, \ldots, x_{k}\right]\right) \wedge n \leq t\right\}
\end{aligned}
$$


- if $k>t$

$$
\begin{aligned}
& \mathcal{T}\left(\left(?\left[x_{1}, \ldots, x_{k}\right]\right)_{t}^{(h)}\right) \\
& =\mathcal{T}\left(?\left[h\left(x_{1}\right)_{t}^{(h)}, \ldots, h\left(x_{k}\right)_{t}^{(h)}\right]\right) \\
& =\{h k\} \cup \mathcal{T}\left(\left(x_{1}\right)_{t}^{(h)}\right) \cup \cdots \cup \mathcal{T}\left(\left(x_{k}\right)_{t}^{(h)}\right) \\
& =\{h k\} \cup \bigcup_{1 \leq i \leq k}\left\{h n \mid n \in \mathcal{T}\left(x_{i}\right) \wedge n>t\right\} \\
& \cup \bigcup_{1 \leq i \leq k}\left\{n \mid n \in \mathcal{T}\left(x_{i}\right) \wedge n \leq t\right\} \\
& =\{h k\} \cup\left\{h n \mid n \in \bigcup_{1 \leq i \leq k} \mathcal{T}\left(x_{i}\right) \wedge n>t\right\} \\
& \cup\left\{n \mid n \in \bigcup_{1 \leq i \leq k} \mathcal{T}\left(x_{i}\right) \wedge n \leq t\right\} \\
& =\left\{h n \mid n \in\left(\{k\} \cup \bigcup_{1 \leq i \leq k} \mathcal{T}\left(x_{i}\right)\right) \wedge n>t\right\} \\
& \cup\left\{n \mid n \in\left(\{k\} \cup \underset{1 \leq i \leq k}{\bigcup} \mathcal{T}\left(x_{i}\right)\right) \wedge n \leq t\right\} \\
& =\left\{h n \mid n \in \mathcal{T}\left(?\left[x_{1}, \ldots, x_{k}\right]\right) \wedge n>t\right\} \\
& \cup\left\{n \mid n \in \mathcal{T}\left(?\left[x_{1}, \ldots, x_{k}\right]\right) \wedge n \leq t\right\}
\end{aligned}
$$

\section{E. Proof of lemma 3}

We first show that, in any $h$-experiment, the size of the multiset associated with a ?-labelled edge is $n$ where $\bar{n}^{h}$ corresponds to the exponential bundle of branches of this edge. By induction on $\mathcal{R}$ :

- If $\mathcal{R}$ has a final $a x, 1, \perp, \otimes$ or $\not 2$-node, the result is immediate by induction hypothesis.

- If $\mathcal{R}$ has a final ? $d$-node, the size of the multiset associated with its conclusion is 1 with $\overline{1}^{h}=1$ (for the other edges we apply the induction hypothesis).

- If $\mathcal{R}$ has a final ?w-node, the size of the multiset associated with its conclusion is 0 with $\overline{0}^{h}=0$ (for the other edges we apply the induction hypothesis).

- If $\mathcal{R}$ has a final ?c-node, we apply the induction hypothesis to the net without this node and we just have to prove the result for the conclusion of the node. We know that the sizes of the labels of the premisses are $m$ and $n$ with corresponding exponential bundles of branches $\bar{m}^{h}$ and $\bar{n}^{h}$, thus the size of the label of the conclusion is $m+n$ and the exponential bundle of this edge (which has strictly less than $h$ elements since $\operatorname{cosize}(\mathcal{R})<h$ ) is precisely $\overline{m+n}^{h}$.

- If $\mathcal{R}$ has two connected components, we apply the induction hypothesis to the components.
- If $\mathcal{R}$ is reduced to a box, by induction hypothesis applied to the experiments $e_{1}, \ldots, e_{h}$ used in the box to build the experiment, we know that the results associated with the premisse of a given ? $p$-node are all of size $n$ where $\bar{n}^{h}$ corresponds to the exponential bundle of branches of this edge. By definition the label of the conclusion of this ? $p$ node is of size $h n$ and $\overline{h n}^{h}$ is precisely the exponential bundle of this edge.

We now show, by induction on $\mathcal{R}$, that if $x$ is the result of an experiment $e$ of $\mathcal{R}$ then $\mathcal{T}(x)$ contains exactly the sizes of the labels associated (by some experiment used to build $e$, either at depth 0 or inside a box) with the ?-labelled edges of $\mathcal{R}$ which are not premisse of a $p p$ or ? $c$-node:

- If $\mathcal{R}$ has a final $a x, 1, \perp, \otimes$ or $\mathcal{P}$-node, the result is immediate by induction hypothesis.

- If $\mathcal{R}$ has a final ? $d$-node, if $e^{\prime}$ is the restriction of $e$ to the subnet without this final node and if $x^{\prime}$ is its result, $x$ is obtained from $x^{\prime}$ by replacing some $y$ by ? $[y]$. As a consequence, $\mathcal{T}(x)=\mathcal{T}\left(x^{\prime}\right) \cup\{1\}$, and we conclude by induction hypothesis.

- If $\mathcal{R}$ has a final ?w-node, if $e^{\prime}$ is the restriction of $e$ to the subnet without this final node and if $x^{\prime}$ is its result, $x$ is obtained from $x^{\prime}$ by adding some ?[]. As a consequence, $\mathcal{T}(x)=\mathcal{T}\left(x^{\prime}\right) \cup\{0\}$, and we conclude by induction hypothesis.

- If $\mathcal{R}$ has a final ?c-node (with conclusion $c$ and premisses $a$ and $b$ ), if $e^{\prime}$ is the restriction of $e$ to the subnet without this final node and if $x^{\prime}$ is its result, $x$ is obtained from $x^{\prime}$ by replacing a pair $? \mu, ? \nu$ (which are the labels $a$ and $b$ ) by ? $(\mu+\nu)$ (which is the label of $c$ ). By induction hypothesis, $\mathcal{T}\left(x^{\prime}\right)=\mathcal{T}_{0} \cup\{m, n\}$ where $m$ is the size of $\mu$ and $n$ is the size of $\nu$, and we have $\mathcal{T}(x)=\mathcal{T}_{0} \cup\{m+n\}$ corresponding to the fact that $a$ and $b$ are no more roots of maximal exponential trees in $\mathcal{R}$ (due to the ?c-node), while $c$ is.

- If $\mathcal{R}$ has two connected components, we apply the induction hypothesis to the components.

- If $\mathcal{R}$ is reduced to a box with $p$ auxiliary doors, if $e_{1}, \ldots, e_{k}$ are the experiments used in the box, $x_{1}, \ldots, x_{k}$ are their results, and ? $\mu_{i}^{j}$ (with $n_{i}^{j}$ the size of $\mu_{i}^{j}$ ) is the label associated by $e_{i}$ to the $j^{\text {th }}$ auxiliary door then, by induction hypothesis, $\mathcal{T}\left(x_{i}\right)=$ $\mathcal{T}_{i} \cup\left\{n_{i}^{1}, \ldots, n_{i}^{p}\right\}$. And we conclude by $\mathcal{T}(x)=$ $\bigcup_{1 \leq i \leq k} \mathcal{T}_{i} \cup\left\{\sum_{1 \leq i \leq k} n_{i}^{1}, \ldots, \sum_{1 \leq i \leq k} n_{i}^{p}\right\}$.

We conclude with lemma 1: if $x$ is an $h$-point of $\llbracket \mathcal{R} \rrbracket$ then it is the result of an $h$-experiment of $\mathcal{R}$. So that $\mathcal{T}(x)$ contains the sizes of the labels of the roots of the maximal exponential trees (by the second part of the proof) which are all the $n$ such that $\bar{n}^{h}$ is an exponential bundle of branches of $\mathcal{R}$ (by the first part of the proof). 